\title{
Analytical Solution for the Lattice Boltzmann Model Beyond Naviers-Stokes
}

\author{
Jianping Meng $^{1}$ and Yonghao Zhang ${ }^{1, *}$ \\ ${ }^{1}$ Department of Mechanical Engineering, University of Strathclyde, Glasgow, \\ G1 1XJ, United Kingdom
}

Received 30 March 2010; Accepted (in revised version) 12 April 2010

Available online 13 July 2010

\begin{abstract}
To understand lattice Boltzmann model capability for capturing nonequilibrium effects, the model with first-order expansion of the equilibrium distribution function is analytically investigated. In particular, the velocity profile of Couette flows is exactly obtained for the D2Q9 model, which shows retaining the first order expansion can capture rarefaction effects in the incompressible limit. Meanwhile, it clearly demonstrates that the D2Q9 model is not able to reflect flow characteristics in the Knudsen layer.
\end{abstract}

AMS subject classifications: 76P05, 82B40

Key words: Lattice Boltzmann method, rarefied gas dynamics, micro flows, Knudsen layer.

\section{Introduction}

Due to rapid development of micro/nano-technologies and modern material processing techniques such as laser fabrication processing and plasma etching $[9,10,12]$, the research interest in rarefied gas dynamics has shifted to low-speed flows under the standard ambient temperature and pressure. For non-equilibrium flows, the linear constitutive relation for stress, which is assumed in the Navier-Stokes equation, is no longer valid. Therefore, kinetic methods or extended hydrodynamic models have to be employed, e.g., the direct simulation Monte Carlo (DSMC) method, and Grad 13 moment model. However, the DSMC simulations is computationally expensive, especially for slow microflows with small Knudsen number. Meanwhile, the direct solution of the Boltzmann equation is still very complex due to the collisional integral. The extended hydrodynamic models are only applicable to the near hydrodynamic regime.

${ }^{*}$ Corresponding author.

URL: http://cms.mecheng.strath.ac.uk/t4/cmsstaffprofile.asp?id=142

Email: jianping.meng@strath.ac.uk (J. P. Meng),yonghao.zhang@strath.ac.uk (Y. H. Zhang) 
The lattice Boltzmann (LB) framework can be served as an alternative computationally efficient method for non-equilibrium gas flows. It was originally developed for hydrodynamics and is proved to be a viable numerical tool $[1,4,5,17,25]$. Compared to the traditional kinetic theory, the LB framework can be efficient since it utilizes a minimal set of velocities in the phase space [5]. Therefore, significant efforts have been devoted to develop or examine the capability of LB models for finite Knudsen number flows, e.g., $[1,2,6,11,13,21,22,24,26-30]$. It was shown that the LB model with discrete velocity set derived from high-order Gauss Hermite quadratures can provide a computationally efficient way of solving the Boltzmann model equation. It can asymptotically recover the Bhatnagar-Gross-Krook (BGK) equation. With the first order approximation of the equilibrium distribution function, it is equivalent to discrete velocity model (DVM) approach of solving the linearized BGK (LBGK) equation [16]. Therefore, the corresponding LB model can capture non-equilibrium effects.

In this work, we will analytically investigate the capability of LB model for nonequilibrium flows. With the first order expansion, the governing equations for distribution function can be great simplified so that they can be solved directly by using available mathematical techniques $[14,19,20]$. In particular, the exact velocity profile of Couette flows will be obtained for the so-called D2Q9 model [18].

\section{Lattice Boltzmann model}

LB models can be constructed by utilizing the Gauss-Hermite quadratures $[7,8,15,23$, 24]. The Boltzmann-BGK equation is

$$
\frac{\partial f}{\partial t}+\xi \cdot \nabla f+g \cdot \nabla_{\xi} f=-\frac{p}{\mu}\left(f-f^{e q}\right),
$$

where $f$ denotes the distribution function; $\xi$, the phase velocity; $p$, the pressure; $g$, the body force; and $\mu$, the gas viscosity. To examine rarefaction effects, it is convenient to use the following non-dimensional variables

$$
\begin{array}{lll}
\hat{\boldsymbol{r}}=\frac{\boldsymbol{r}}{L^{\prime}}, & \hat{\boldsymbol{u}}=\frac{\boldsymbol{u}}{\sqrt{R T_{0}}}, & \hat{\boldsymbol{t}}=\frac{\sqrt{R T_{0}} t}{L}, \\
\hat{\boldsymbol{g}}=\frac{L \boldsymbol{g}}{R T_{0}}, & \hat{\boldsymbol{\xi}}=\frac{\xi}{\sqrt{R T_{0}}}, & \hat{T}=\frac{T}{T_{0}},
\end{array}
$$

where $u$ is the macroscopic velocity; $R$, the gas constant; $T$, the gas temperature; $T_{0}$, the reference temperature; $r$, the spatial position; and $L$, the characteristic length of the flow system. The symbol hat, which denotes dimensionless value, will hereinafter be omitted. The Knudsen number can be defined by using macroscopic properties as

$$
K n=\frac{\mu \sqrt{R T_{0}}}{p L} .
$$


Based on these non-dimensional variables, the non-dimensional form of the BGK equation becomes

$$
\frac{\partial f}{\partial t}+\xi \cdot \nabla f+g \cdot \nabla_{\xi} f=-\frac{1}{K n}\left(f-f^{e q}\right),
$$

where the Maxwell distribution in D-dimensional Cartesian coordinates can be written as

$$
f^{e q}=\frac{\rho}{(2 \pi T)^{D / 2}} \exp \left[\frac{-(\xi-u)^{2}}{2 T}\right]
$$

By choosing an apropriate Hermite expansion and a sufficiently accurate Gauss-Hermite quadrature, Eq. (2.4) can be discretized as

$$
\frac{\partial f_{\alpha}}{\partial t}+\xi_{\alpha} \cdot \nabla f_{\alpha}=-\frac{1}{K n}\left(f_{\alpha}-f_{\alpha}^{e q}\right)+g_{\alpha}
$$

where

$$
f_{\alpha}=\frac{w_{\alpha} f\left(\boldsymbol{r}, \boldsymbol{\xi}_{\alpha}, t\right)}{\omega\left(\boldsymbol{\xi}_{\alpha}\right)}, \quad f_{\alpha}^{e q}=\frac{w_{\alpha} f^{e q}\left(\boldsymbol{r}, \boldsymbol{\xi}_{\alpha}, t\right)}{\omega\left(\boldsymbol{\xi}_{\alpha}\right)}, \quad \text { and } \quad g_{\alpha}=\frac{w_{\alpha} F\left(\boldsymbol{r}, \boldsymbol{\xi}_{\alpha}, t\right)}{\omega\left(\boldsymbol{\xi}_{\alpha}\right)} .
$$

Therefore, the LB equation, i.e., Eq. (2.6) is now obtained by discretizing Eq. (2.4) in the velocity space. The specific form of $f_{\alpha}^{e q}$ and $g_{\alpha}$ can be accordingly obtained. For isothermal problems where $T=1$, a typical choice is the following second order form

$$
\begin{aligned}
& f_{\alpha}^{e q}=w_{\alpha} \rho\left\{1+\boldsymbol{\xi}_{\alpha} \cdot \boldsymbol{u}+\frac{1}{2}\left[\left(\boldsymbol{\xi}_{\alpha} \cdot \boldsymbol{u}\right)^{2}-u^{2}\right]\right\}, \\
& g_{\alpha}=w_{\alpha} \rho\{\boldsymbol{g} \cdot \boldsymbol{\xi}+(\boldsymbol{g} \cdot \boldsymbol{\xi})(\boldsymbol{u} \cdot \boldsymbol{\xi})-\boldsymbol{g} \cdot \boldsymbol{u}\} .
\end{aligned}
$$

It was shown that the Gauss-Hermite quadrature is the upmost factor in determining how accurate rarefaction effects can be captured while the Hermite expansion order for the equilibrium distribution function is not relevant [16]. The first-order expansion is sufficient for the LB model to capture isothermal non-equilibrium flow phenomena in the incompressible limit, if the Gauss-Hermite quadratures can provide sufficient accuracy [16]. This has been confirmed both theoretically and numerically [16].

In this work, we will further analytically investigate simple Couette flows to see whether the rarefaction effect can be described in the LB model. The equilibrium distribution function, $f^{e q}$, where the first order expansion is retained, can be written as

$$
f_{\alpha}^{e q}=w_{\alpha} \rho\left\{1+\xi_{\alpha} \cdot \boldsymbol{u}\right\}
$$

By introducing

$$
f_{\alpha}=f_{\alpha}^{0}\left(1+\varphi_{\alpha}\right)
$$

where $f_{\alpha}^{0}$ is the equilibrium distribution function with $u=0$, Eq. (2.6) can rewritten as (see [16])

$$
\frac{\partial \varphi_{\alpha}}{\partial t}+\boldsymbol{\xi} \cdot \nabla \varphi_{\alpha}=-\frac{1}{K n}\left(\varphi_{\alpha}-\xi_{\alpha} \cdot \boldsymbol{u}\right)
$$


in the incompressible limit, which is the linearized form of Eq. (2.6). For simplicity, the force term has been omitted. In the following, the so-called D2Q9 model will be examined, which has the discrete velocity set as

$$
\begin{aligned}
& \xi_{\alpha x}=[0, \sqrt{3}, \sqrt{3}, 0,-\sqrt{3},-\sqrt{3},-\sqrt{3}, 0, \sqrt{3}], \\
& \xi_{\alpha y}=[0,0, \sqrt{3}, \sqrt{3}, \sqrt{3}, 0,-\sqrt{3},-\sqrt{3},-\sqrt{3}], \\
& w_{\alpha}=\left[\frac{4}{9}, \frac{1}{9}, \frac{1}{36}, \frac{1}{9}, \frac{1}{36}, \frac{1}{9}, \frac{1}{36}, \frac{1}{9}, \frac{1}{36}\right] .
\end{aligned}
$$

\section{Analytical solution for the Couette flow}

With simple geometry, the Couette flow can mimic many realistic shear dominant applications. To ensure the rarefaction effects are not discounted by inappropriate boundary condition, the Maxwellian diffuse reflection boundary condition will be used [3] to describe gas/surface interactions. The lower plate is located at $y=-1 / 2$ and the upper one at $y=1 / 2$, and they are set to be moving with the velocities $U_{1}$ and $U_{2}$ respectively.

For steady Couette flow, the exact form of nine governing equations for the D2Q9 model can be written as

$$
\begin{aligned}
& \omega \varphi_{1}(y)=0 \\
& -\omega \sqrt{3} u_{x}+\omega \varphi_{2}(y)=0 \\
& \sqrt{3} \frac{d}{d y} \varphi_{3}(y)-\omega \sqrt{3} u_{x}+\omega \varphi_{3}(y)=0 \\
& \sqrt{3} \frac{d}{d y} \varphi_{4}(y)+\omega \varphi_{4}(y)=0 \\
& \sqrt{3} \frac{d}{d y} \varphi_{5}(y)+\omega \sqrt{3} u_{x}+\omega \varphi_{5}(y)=0 \\
& \omega \sqrt{3} u_{x}+\omega \varphi_{6}(y)=0 \\
& -\sqrt{3} \frac{d}{d y} \varphi_{7}(y)+\omega \sqrt{3} u_{x}+\omega \varphi_{7}(y)=0 \\
& -\sqrt{3} \frac{d}{d y} \varphi_{8}(y)+\omega \varphi_{8}(y)=0 \\
& -\sqrt{3} \frac{d}{d y} \varphi_{9}(y)-\omega \sqrt{3} u_{x}+\omega \varphi_{9}(y)=0
\end{aligned}
$$

where

$$
\omega=\frac{1}{K n} .
$$

To obtain the velocity profile, only Eqs. (3.1b)-(3.1i) are relevant, though one can directly solve Eqs. (3.1a),(3.1d) and (3.1h). Moreover, we have the following relations

$$
\varphi_{6}(y)=-\varphi_{2}(y)
$$




$$
\begin{aligned}
& \sqrt{3} \frac{d}{d y}\left[\varphi_{3}(y)+\varphi_{5}(y)\right]+\omega\left[\varphi_{3}(y)+\varphi_{5}(y)\right]=0, \\
& -\sqrt{3} \frac{d}{d y}\left[\varphi_{7}(y)+\varphi_{9}(y)\right]+\omega\left[\varphi_{7}(y)+\varphi_{9}(y)\right]=0 .
\end{aligned}
$$

Therefore, there are only four independent equations.

One can explicitly solve Eqs. (3.1b)-(3.1i). The solutions are

$$
\begin{aligned}
& \varphi_{2}(y)= \frac{1}{4 \omega}\left(\omega C_{2} \mathrm{e}^{\frac{1}{3} \omega \sqrt{3} y}+2 C_{4} y \omega+2 C_{3} \omega+4 \sqrt{3} C_{4}\right)+\frac{1}{2} C_{4} y \\
& \quad+\frac{1}{2} C_{3}-\frac{1}{4} C_{2} \mathrm{e}^{\frac{1}{3} \omega \sqrt{3} y}, \\
& \varphi_{3}(y)= \frac{1}{2} C_{1} \mathrm{e}^{-\frac{1}{3} \omega \sqrt{3} y}+C_{4} y+C_{3}, \\
& \varphi_{5}(y)= \frac{1}{2} C_{1} \mathrm{e}^{-\frac{1}{3} \omega \sqrt{3} y}-C_{4} y-C_{3}, \\
& \varphi_{6}(y)=-\frac{1}{4 \omega}\left(\omega C_{2} \mathrm{e}^{\frac{1}{3} \omega \sqrt{3} y}+2 C_{4} y \omega+2 C_{3} \omega+4 \sqrt{3} C_{4}\right) \\
& \quad \quad-\frac{1}{2} C_{4} y-\frac{1}{2} C_{3}+\frac{1}{4} C_{2} \mathrm{e}^{\frac{1}{3} \omega \sqrt{3} y}, \\
& \varphi_{7}(y)=C_{2} \mathrm{e}^{\frac{1}{3} \omega \sqrt{3} y}-\frac{1}{2 \omega}\left(\omega C_{2} \mathrm{e}^{\left.\frac{1}{3} \omega \sqrt{3} y+2 C_{4} y \omega+2 C_{3} \omega+4 \sqrt{3} C_{4}\right),}\right. \\
& \varphi_{9}(y)=\frac{1}{2 \omega}\left(\omega C_{2} \mathrm{e}^{\frac{1}{3} \omega \sqrt{3} y}+2 C_{4} y \omega+2 C_{3} \omega+4 \sqrt{3} C_{4}\right),
\end{aligned}
$$

therefore, the macroscopic velocity $u_{x}=\sum_{\alpha} w_{\alpha} \xi_{\alpha x} \varphi_{\alpha}$ is

$$
u_{x}=\frac{1}{3 \omega}\left(\sqrt{3} C_{4} y \omega+\sqrt{3} C_{3} \omega+3 C_{4}\right) .
$$

The coefficients can be determined by the kinetic boundary conditions, i.e.,

$$
\begin{aligned}
C_{1} & =0, & C_{2} & =0, \\
C_{3} & =\frac{1}{2} \frac{\sqrt{3}\left(\omega U_{1}+4 \sqrt{3} U_{1}+U_{2} \omega\right)}{\omega+2 \sqrt{3}}, & C_{4} & =\frac{\omega \sqrt{3}\left(-U_{1}+U_{2}\right)}{\omega+2 \sqrt{3}} .
\end{aligned}
$$

Finally, we obtain the macroscopic velocity profile across the two plates for different Knudsen numbers

$$
u_{x}=\frac{y \Delta U}{1+2 \sqrt{3} K n}+U
$$

where

$$
U=\frac{U_{1}+U_{2}}{2}, \text { and } \Delta U=U_{2}-U_{1} .
$$

This solution completely agrees with the one given in $[2,28]$. However, it is interesting to compare our analytical process with the one used in $[2,28]$ where the second-order expansion for the equilibrium distribution function is used. This again clearly proves 
that retaining the first-order expansion of the equilibrium distribution function is sufficient for capturing rarefaction effects in the incompressible limit. The result shows that the D2Q9 model fails to capture flow characteristics in the Knudsen layer, i.e., the nonlinear constitutive relations. Our analysis will be extended to search for exact solutions to higher-order LB models, and we will report our findings in due course.

\section{Conclusions}

We have analytically investigated the capability of LB model with the first-order expansion of the equilibrium distribution function for non-equilibrium flows. The exact velocity profile of Couette flow for the D2Q9 model reveals that the LB model with the first-order expansion of the equilibrium distribution function is sufficient for capturing rarefaction effects in the incompressible limit.

\section{Acknowledgments}

This work was financially supported by the Engineering and Physical Sciences Research Council U.K. under Grants No. EP/ F028865/1. The research leading to these results has received the funding from the European Community's Seventh Framework Programme FP7/2007-2013 under grant agreement ITN GASMEMS No. 215504.

\section{References}

[1] C. K. Aidun And J. R. Clausen, Lattice-Boltzmann method for complex flows, Annu. Rev. Fluid. Mech., 42 (2010), pp. 439-472.

[2] S. Ansumali, I. V. Karlin, S. Arcidiacono, A. Abbas and N. I. Prasianakis, Hydrodynamics beyond Navier-Stokes: Exact solution to the lattice Boltzmann hierarchy, Phys. Rev. Lett., 98(4) (2007), 124502.

[3] S. Ansumali And I. V. Karlin, Kinetic boundary conditions in the lattice Boltzmann method, Phys. Rev. E., 66(6) (2002), 026311.

[4] R. Benzi, S. Succi AND M. Vergassola, The lattice Boltzmann equation: Theory and applications, Phys. Rep., 222 (1992), pp. 145-197.

[5] S. Y. CHEN AND G. D. Doolen, Lattice Boltzmann method for fluid flows, Annu. Rev. Fluid. Mech., 30 (1998), pp. 329-364.

[6] Z. L. GUO, T. S. ZHAO AND Y. SHI, Physical symmetry, spatial accuracy, and relaxation time of the lattice Boltzmann equation for microgas flows, J. Appl. Phys., 99(8) (2006), 074903.

[7] X. Y. He AND L. S. LUO, A priori derivation of the lattice Boltzmann equation, Phys. Rev. E., 55 (1997), pp. 6333-6336.

[8] X. Y. HE AND L. S. LUO, Theory of the lattice Boltzmann method: From the Boltzmann equation to the lattice Boltzmann equation, Phys. Rev. E., 56 (1997), pp. 6811-6817.

[9] C. M. HO AND Y. C. TAI, Review: MEMS and its applications for flow control, J. Fluid. Eng., 118 (1996), pp. 437-447.

[10] C. M. Ho AND Y. C. TAI, Micro-electro-mechanical-systems (MEMS) and fluid flows, Annu. Rev. Fluid. Mech., 30 (1998), pp. 579-612. 
[11] I. V. Karlin AND S. Ansumali, Renormalization of the lattice Boltzmann hierarchy, Phys. Rev. E., 76(4) (2007), 025701.

[12] G. KARniadAKIS, A. BeSKOK AND N. AluRU, Microflows and Nanoflows: Fundamentals and Simulation (Interdisciplinary Applied Mathematics), Springer, 2005.

[13] S. H. Kim, H. PiTSCH AND I. D. BOYD, Accuracy of higher-order lattice Boltzmann methods for microscale flows with finite Knudsen numbers, J. Comput. Phys., 227 (2008), pp. 86558671.

[14] L. S. LuO, Analytic solutions of linearized lattice Boltzmann equation for simple flows, J. Stat. Phys., 88 (1997), pp. 913-926.

[15] N. S. MARTYS, X. W. SHAN AND H. D. CHEN, Evaluation of the external force term in the discrete Boltzmann equation, Phys. Rev. E., 58 (1998), pp. 6855-6857.

[16] J. P. MeNG AND Y. H. ZHANG, Accuracy analysis of high-order lattice Boltzmann models for rarefied gas flows, http://arxiv. org/abs/0908.4520, 2009.

[17] Y. H. QIAN, S. SUCCI AND S. ORSZAG, Recent advances in lattice Boltzmann computing, Annu. Rev. Comput. Phys., III (1995), pp. 192-242.

[18] Y. H. QIAN, D. D'HumièRES AND P. LALLEMAND, Lattice BGK models for Navier-Stokes equation, Europhys. Lett., 17 (1992), pp. 479-484.

[19] J. R. SARAF, Temperature jump problem in the Knudsen layer using the Bhatnagar-Gross-Krook model, Acta. Mech., 9 (1970), pp. 341-345.

[20] J. R. SARAF, Heat transfer in rarefied couette flow on the basis of discrete ordinate method, Appl. Sci. Res., 24 (1971), pp. 467-476.

[21] M. SBRAGAGLIA AND S. SUCCI, Analytical calculation of slip flow in lattice Boltzmann models with kinetic boundary conditions, Phys. Fluids., 17(8) (2005), 093602.

[22] M. SBRAGagLia AND S. SUCCI, A note on the lattice Boltzmann method beyond the ChapmanEnskog limits, Europhys. Lett., 73 (2006), pp. 370-376.

[23] X. W. SHAN AND X. Y. HE, Discretization of the velocity space in the solution of the Boltzmann equation, Phys. Rev. Lett., 80 (1998), pp. 65-68.

[24] X. W. SHAN, X. F. YUAN AND H. D. CHEN, Kinetic theory representation of hydrodynamics: A way beyond the Navier Stokes equation, J. Fluid. Mech., 550 (2006), pp. 413-441.

[25] S. SuCci, I. V. Karlin And H. D. Chen, Colloquium: Role of the $H$ theorem in lattice Boltzmann hydrodynamic simulations, Rev. Mod. Phys., 74 (2002), pp. 1203-1220.

[26] G. H. TANG, Y. H. ZHANG AND D. R. EMERSON, Lattice Boltzmann models for nonequilibrium gas flows, Phys. Rev. E., 77(6) (2008), 046701.

[27] F. TOSCHI AND S. SUCCI, Lattice Boltzmann method at finite Knudsen numbers, Europhys. Lett., 69 (2005), pp. 549-555.

[28] W. P. YUdistiaWAN, S. Ansumali AND I. V. Karlin, Hydrodynamics beyond NavierStokes: The slip flow model, Phys. Rev. E., 78(14) (2008), 016705.

[29] Y. H. ZHANG, R. S. QIN AND D. R. EMERSON, Lattice Boltzmann simulation of rarefied gas flows in microchannels, Phys. Rev. E., 71(4) (2005), 047702.

[30] Y. H. Zhang, X. J. Gu, R. W. Barber and D. R. Emerson, Capturing Knudsen layer phenomena using a lattice Boltzmann model, Phys. Rev. E., 74(7) (2006), 046704. 